\title{
Effect of sodium dodecyl sulfate on flow and electrokinetic properties of Na-activated bentonite dispersions
}

\author{
E GÜNISTER, S İşÇİ, A ALEMDAR and N GÜNGÖR* \\ Department of Physics, Istanbul Technical University, 34469, Maslak, Istanbul, Turkey
}

MS received 19 August 2003; revised 6 February 2004

\begin{abstract}
The present study reports the effect of anionic surfactant sodium dodecyl sulfate (SDS, $\mathrm{C}_{12} \mathrm{H}_{25}$ $\mathrm{OSO}_{3} \mathrm{Na}$ ) upon the electrokinetic (electrophoretic mobility, zeta potential) and rheological (viscosity, yield value) properties of the Ca-bentonitic clay found in Turkey and its Na-activated form. The SDS dispersant was added in different concentrations in the range of $1 \times 10^{-5}-5 \times 10^{-2} \mathrm{~mol} / \mathrm{l}$. The results show that the viscosity and zeta potential values of bentonite dispersion are affected by the addition of anionic surfactant. The obtained data are analysed by considering the kind of exchangeable cations. Thixotropic property effect was observed in bentonite dispersions.
\end{abstract}

Keywords. Bentonite; sodium dodecyl sulfate; anionic surfactant; rheology; zeta potential.

\section{Introduction}

Bentonite, which is a kind of smectite group clay mineral, is almost made up of $80 \%$ of montmorillonite and contains a crystal lattice with three layers. It is an important clay having a variety of uses because of its colloidal property when it is mixed with water, swelling in water and in some organo environment, and having high plasticity. The industrial application of bentonite dispersions is very widespread. Bentonite with its higher ion exchange capacity and surface area is used as an industrial raw material in sorptive, catalytic and rheological applications. They are used in different branches of industry, such as in drilling fluids, dyes, pharmaceutical applications, paper, cement and ceramics.

The layer structure of bentonite and its good ionexchange capacity are mainly responsible for imparting gelation and in turn relatively high viscosity in bentonite. Bentonite dispersions have the advantage over other clays in being very sensitive to surfactants, polymers and electrolytes. Cationic and anionic surfactants greatly influence the flow behaviour and electrokinetic properties of bentonite dispersions. Extensive research has been carried out on the rheological and electrokinetic properties of bentonite dispersions (Welzen et al 1981; Lagaly 1989; Luckham and Rossi 1999; Güngör 2000; Güngör et al 2001; Yalçin et al 2002a, b; Alemdar et al 2003).

The aim of this two-phase study is first to determine and compare the rheological properties of two bentonitic clay samples, and secondly, to determine the changes in rheological and electrokinetic properties by SDS addi-

\footnotetext{
*Author for correspondence
}

tions at different concentrations and to evaluate the results by considering the interaction modes between the particles and surfactant molecules.

\section{Experimental}

The clay sample was obtained from the bentonite deposits in Edirne-Lalapasa, Turkey (courtesy, Bensan Co.). Samples were taken from three sections at random locations. A Philips PW 1040 model X-ray diffractometer instrument was used to determine the clay mineral types. The dominant clay mineral was found to be dioctahedral montmorillonite $(98 \%)$ with minor amounts of illite $(0 \cdot 5-$ $1 \%)$, calcite (1-2\%), feldspar (1\%) and quartz (0.2\%).

The natural sample has been identified as Ca-bentonite. The Na-activated bentonite was obtained from natural bentonite (35\% humidity) by treating the clay with $4 \%(\mathrm{w} / \mathrm{v})$ $\mathrm{NaHCO}_{3}$ solution. Natural bentonite is labelled CaLB and activated clay as NaLB.

The chemical composition of the samples was determined by atomic absorption spectroscopy (Perkin Elmer 3030 model) except for silica, which was determined gravimetrically. Chemical analysis of the clays is given in table 1 .

A stock dispersion was prepared by mixing $4 \mathrm{~g}$ of bentonite with $100 \mathrm{ml}$ water and shaken for $24 \mathrm{~h}$. Aliquots of this solution were mixed with aliquots of surfactant solutions so that the solid content of the dispersions was $2 \%$ in the rheological experiments. The dispersions were shaken for $24 \mathrm{~h}$ after mixing.

All rheological measurements of the clay-surfactant dispersions were performed at their ambient $\mathrm{pH}$ values. The natural $\mathrm{pH}$ values of $\mathrm{CaLB}$ and NaLB dispersions were found to be 8 and 10 , respectively. 
Rheological properties such as viscosity, shear rate $(\mathrm{d} v / \mathrm{d} r)$ and shear stress $(\tau)$ were measured using a Brookfield DVIII + type low-shear viscometer for bentonite suspensions before and after surfactants treatment at room temperature. Flow curves were analysed using the Bingham model, where the Bingham yield stress, $\tau$, is given by the relation

$$
\tau=\tau_{\mathrm{e}}+\eta_{\mathrm{p} 1} \gamma
$$

Here $\tau_{\mathrm{e}}$ is the extrapolated yield value and $\eta_{\mathrm{pl}}$ the plastic viscosity. Once the curve of shear stress vs shear rate was obtained for $\mathrm{CaLB}$ and $\mathrm{NaLB}$ dispersions under the given experimental conditions, the value of the extrapolated shear stress was determined from the intersection of the extrapolated linear portion of the curve with the axis. The method of least squares was used to determine the values of this intersection. Plastic viscosity was derived from the slope of this linear section. The apparent viscosity of a nonNewtonian dispersion is the shear stress/shear rate ratio at any shear of rate.

A stable colloidal dispersion coagulates when a certain amount of salt is added. The minimum salt concentration that is needed to cause coagulation of a colloidal dispersion is called critical coagulation concentration, $c_{\mathrm{K}}$. The critical concentration of the organic salts (surfactant) was determined by visual inspection of the behaviour of bentonite dispersions. For this purpose, diluted $(0 \cdot 25 \% \mathrm{w} / \mathrm{w})$ bentonite dispersions were prepared at different SDS concentrations in test tubes at room temperature. The minimum salt concentration that coagulates the dispersion was recorded as critical coagulation concentration. The $c_{\mathrm{K}}$ value of NaLB was found to be $7 \cdot 10^{-3} \mathrm{~mol} / \mathrm{l}$.

Zeta potential measurements were carried out using a Malvern Instrument, Zetasizer 2000. The optic unit contains a $5 \mathrm{~mW} \mathrm{He}-\mathrm{Ne}(638 \mathrm{~nm})$ laser. In this instrument, to make an electrophoretic mobility measurement, laser beams are made to cross at a particular point in the cell. Particles in the cell were illuminated by these beams. At the crossing point of the beams, Young's interference fringes were formed. Particles moving through the fringes under the influence of the applied electric field scatter light whose intensity fluctuates with a frequency that is related to the particle velocity. The photons detected by photomultiplier are fed to a digital correlator, the resulting function being analysed to determine the frequency spectrum, from which the mobility and hence the zeta potential are calculated. The relation between ele- ctrophoretic mobility and zeta potential is given by the Schmoluchowski formula:

$$
\mu_{\mathrm{E}}=\frac{\varepsilon_{\mathrm{r}} \varepsilon_{\mathrm{o}} \zeta}{\eta},
$$

where $\mu_{\mathrm{E}}$ is the electrophoretic mobility, $\eta$ the liquid viscosity and $\varepsilon_{\mathrm{r}}$ and $\varepsilon_{\mathrm{o}}$ are the dielectric constants of the medium and free spaces, respectively.

Zeta potential was measured, injecting a small portion of the dispersion into the cell of Zetasizer 2000 instrument at $25^{\circ} \mathrm{C}$. To be able to obtain correct concentration interval, which are recommended for the apparatus before the measurements, all the dispersions were centrifuged at $4500 \mathrm{rpm}$ for $30 \mathrm{~min}$ and then supernatants were used for zeta potential measurements. Multiple measurements of the electrophoretic mobility of the particles were made. SDS is an anionic surfactant (from Fluka Chemical, Switzerland) soluble in water and its molecular weight is $288 \cdot 3 \mathrm{~g} / \mathrm{mol}$.

\section{Results}

The effect of surfactants on clay dispersions is dependent on the differences in the experimental conditions such as the particle size, shape, surface charge of clay particles, the degree of dispersion, nature of exchangeable cations, $\mathrm{pH}$ of dispersions and pretreatment reactions (Michaels and Morelos 1955; Welzen et al 1981; Penner and Lagaly 2000; Janek and Lagaly 2003).

The plastic viscosity values of CaLB and NaLB have been found to be 1.75 and $2.78 \mathrm{mPas}$, respectively. This difference is caused by the change in swelling and dispersion behaviours of the neutral bentonite after the activation process. Exchangeable cations, which determine orientation of many physical properties of bentonitic clays, such as particle size, surface charge, surface area and particle number per unit volume, also have a strong influence on the dispersion mechanisms. The increase of viscosity and consistency of Ca-bentonite dispersions after addition of sodium ion is a direct consequence of the opposite effect of $\mathrm{Na}^{+}$and $\mathrm{Ca}^{++}$ions. When clays are dispersed in water, Na-activated bentonite has unique crystal layers in very fine colloidal particles from tens to hundred micrometers, because the electrostatic attraction among the crystal layers is weaker. On the other hand, for the Ca-bentonite, the mean particle size is kept at several $\mu \mathrm{m}$ 's in water, because the electrostatic attraction among the crystal layers is stronger.

Table 1. Chemical analyses (wt\%) of bentonite samples.

\begin{tabular}{lllllllll}
\hline & $\mathrm{SiO}_{2}$ & $\mathrm{Al}_{2} \mathrm{O}_{3}$ & $\mathrm{CaO}$ & $\mathrm{Na}_{2} \mathrm{O}$ & $\mathrm{MgO}$ & $\mathrm{Fe}_{2} \mathrm{O}_{3}$ & $\mathrm{~K}_{2} \mathrm{O}$ & $\mathrm{TiO}_{2}$ \\
\hline Natural bentonite & $61 \cdot 06$ & 19.91 & $3 \cdot 12$ & $0 \cdot 01$ & $5 \cdot 27$ & $2 \cdot 26$ & $0 \cdot 20$ & $0 \cdot 18$ \\
Na-activated bentonite & $56 \cdot 77$ & $19 \cdot 27$ & $3 \cdot 5$ & $4 \cdot 54$ & 4.48 & $2 \cdot 33$ & $0 \cdot 41$ & $0 \cdot 22$ \\
\hline
\end{tabular}


The adsorption phenomena of anionic surfactant SDS on bentonite particles as a function of concentration of the surfactant is schematically presented in figure 1 . When clays are added into water, colloidal clay particles begin to swell and disperse in suspension. Clay particles move randomly in the dispersion, and this motion, called Brownian motion, causes particle-to-particle collisions. When clay minerals approach a sufficient short distance, different interactions among clay particles will begin, and Coulombic and Van der Waals forces play an important role. Dispersion of clay particles flocculates in three possible modes of particle association (Olphen 1977): (i) association between flat oxygen planes of two parallel platelets (face-to-face, F/F), (ii) association between edge surface of neighbouring particles (edge-to-edge, E/E) and (iii) association between edge surfaces and flat oxygen planar surface (edge-to-face, E/F).

When anionic surfactant is slowly added into dispersion, negatively charged hydrophilic heads bond with positively charged edges of clay minerals (figure $1 \mathrm{~b}$ ). This will decrease the electrostatic attraction between clay particles and might destroy some possible EF type contacts between bentonite particles. At higher surfactant concentration, free hydrophilic edges come together to develop bridge flocculation; consequently, the viscosity values increase (figure 1c). Then a decrease in viscosity with further addition of surfactant corresponds to the full coverage of the particles. This decrease of the viscosity is related to the steric push occurring between SDS covered clay particles (figure 1d).

The shear stress $(\tau)$ vs shear rate $(\gamma)$ curve for a $2 \% \mathrm{w} / \mathrm{w}$ of CaLB and NaLB bentonite dispersions is shown in figure 2. The suspensions of the sample exhibit nearly Newtonian behaviour. The viscosity of a Newtonian dispersion is the shear stress/shear rate ratio.

In figure 3 , the plastic viscosity of bentonite dispersions is plotted as a function of increasing SDS concen- tration. It can be observed that the surfactant is more effective for NaLB dispersions while the rheological properties of CaLB dispersions are least affected.

Plastic viscosity of NaLB dispersions increases slightly on addition of a small amount of SDS. Surfactant anions are adsorbed on the edges and compensated the positive charges. Some edge/face contacts are destroyed. As seen from the figure, the initial plastic viscosity value of the dispersion remains nearly constant until the concentration reaches $5 \times 10^{-4} \mathrm{SDS}$. With further addition of SDS, the viscosity value increases and it shows relatively higher resistance against the flow. The increase corresponds to an increasing amount of adsorbed SDS anions. The particles are linked by interpenetrating alkyl chains, which are responsible for the increase in the viscosity value to a maximum. For NaLB dispersions, at the higher SDS surfactant concentration a drop in the viscosity is observed. This decrease of viscosity at still higher SDS concentration indicates recharging of the edges by SDS anions, which are adsorbed together with their counterions.

The zeta potential values were $-11 \mathrm{mV}$ and $-36.5 \mathrm{mV}$ for CaLB and NaLB, respectively. The zeta potential value changed in a similar way as a function of surfactant concentration for NaLB and CaLB bentonite dispersions (figure 4). The addition of SDS into the dispersions neutralizes the positive edge charges of the bentonite particles. This results in an increase in the total negative charge of the particles. After the SDS concentration of $10^{-3} \mathrm{~mol} / \mathrm{l}$, electrostatic repulsion between the particles becomes higher and dispersions showed higher zeta potential values. The curves indicate a deflocculant system. This result correlates with the trend of viscosity values after the addition of the additives (figure 3 ). The increase in the plastic viscosity of NaLB dispersion after surfactant concentration of $10^{-3} \mathrm{~mol} / \mathrm{l}$ is the result of the interaction between alkyl chains of SDS molecules, which were attached on the clay surface.

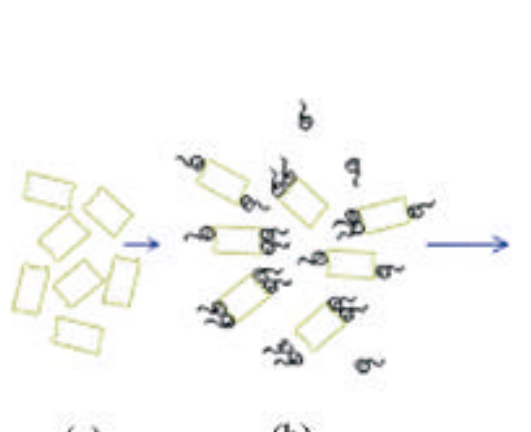

(a)

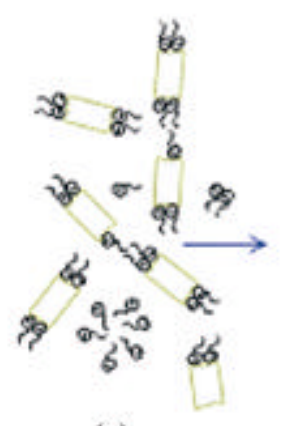

(c)

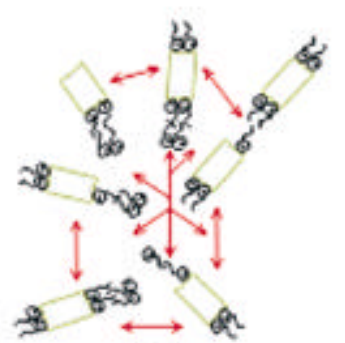

(d)

\section{Interaction of anionic surfactant (SDS) with clay particles}

Figure 1. Schematic representation of adsorption of anionic surfactant on bentonite particles as a function of the concentration of surfactant. 
The flow model of the CaLB did not change by SDS addition. After SDS addition, the NaLB displayed a Bingham flow model (Güven 1992). Figure 5 shows the effect of SDS on the flow behaviour of bentonite dispersion $(2 \% \mathrm{w} / \mathrm{w})$ at the surfactant concentration of $5 \times 10^{-2} \mathrm{~mol} / \mathrm{l}$. Increasing SDS concentration resulted in increasing shear stress in
NaLB dispersion. The results shown in figure 5 clearly show an increase in gelation on addition of SDS for Na-activated bentonite dispersion. This gelation was considered to be due to interaction between alkyl chains of SDS. The gel state is characterized by the appearance of the yield value. The gel-like dispersion showed pronounced thixotropy.

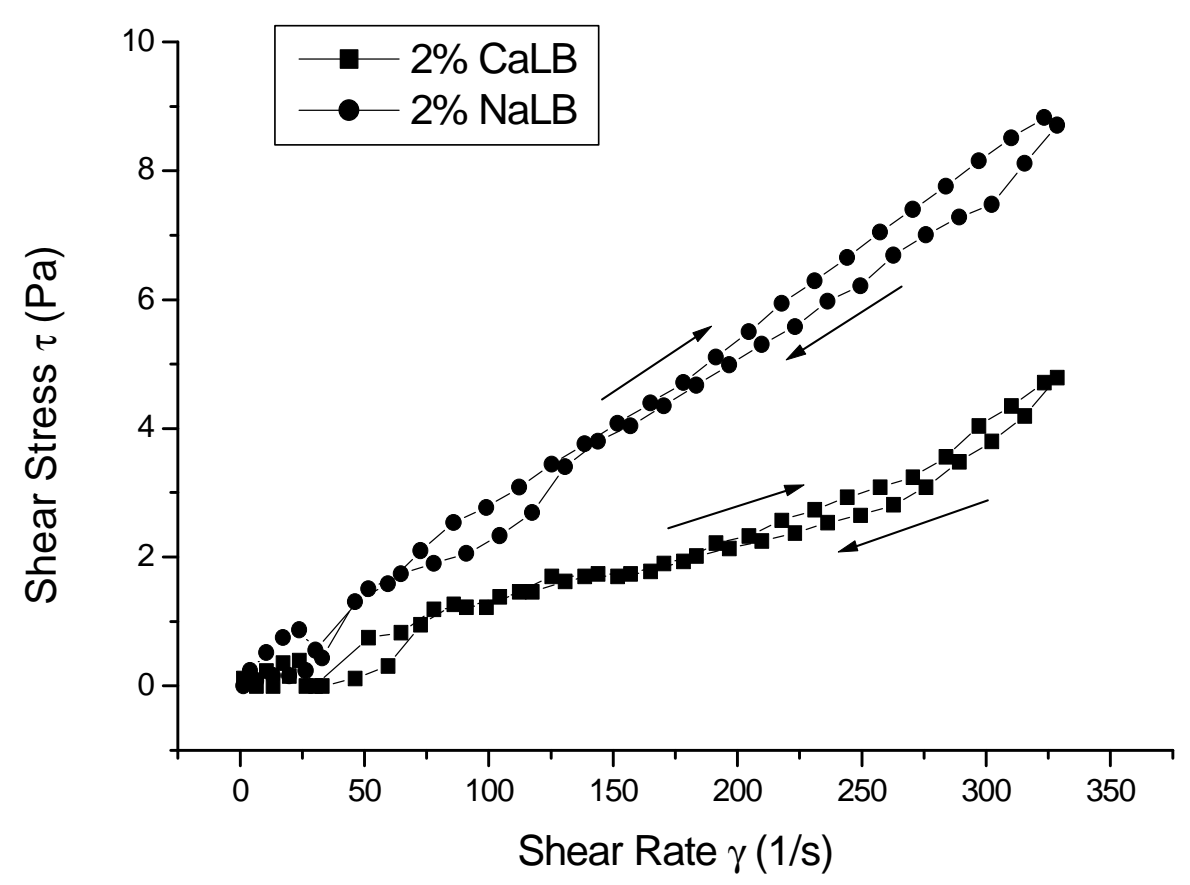

Figure 2. The shear stress $(\tau)$ vs shear rate $(\gamma)$ rheogram of CaLB and NaLB dispersions.

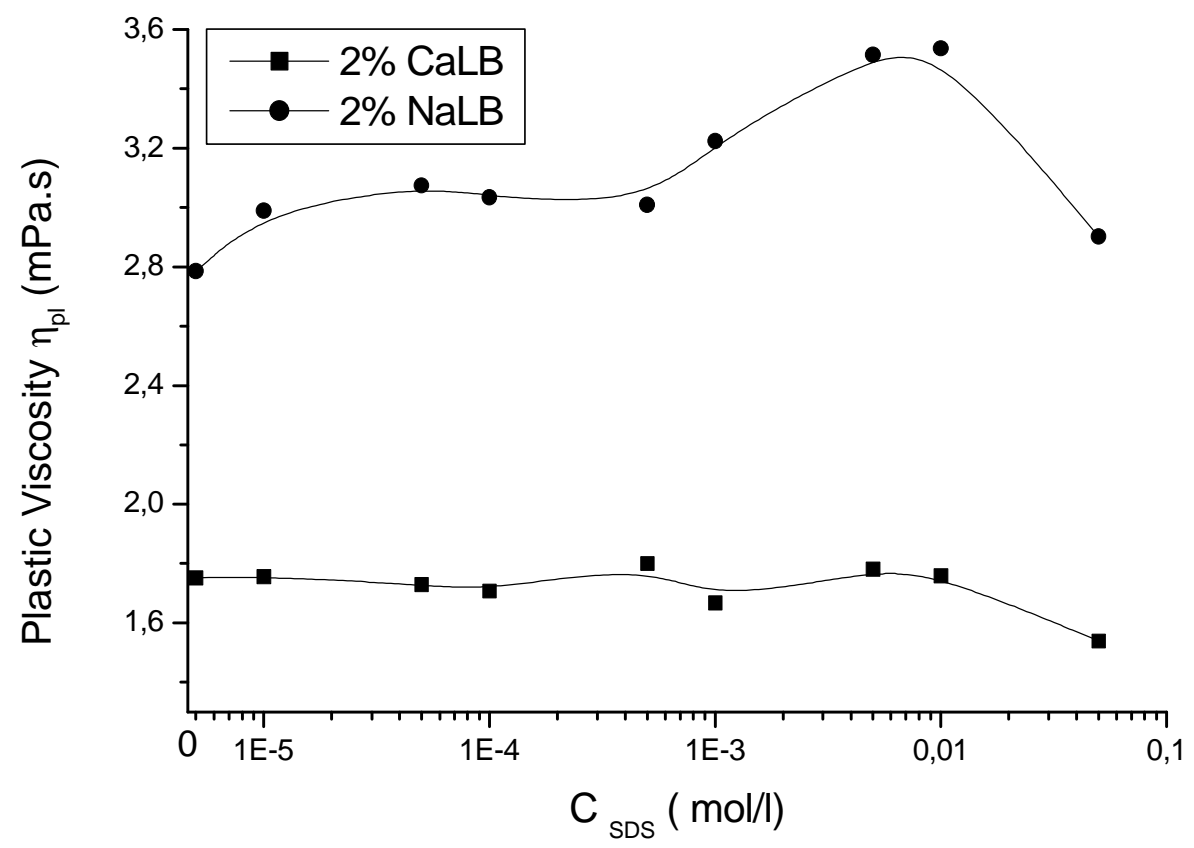

Figure 3. The plastic viscosity of natural and Na-activated bentonite as a function of SDS concentrations. 
Formation of the gel depends on the particle-particle interactions, which are governed by the type and concentration of the surfactants, on the solid content of clay particles and the shape and size of the particles. From a rheological point of view, thixotropy is defined as iso- thermal and reversible gel-liquid transformation upon mechanical agitation (Güven 1992). A thixotropic system begins to flow on stirring and thickens again when left undisturbed. In other words, thixotropy is a time dependent phenomenon. The network structure of the semirigid

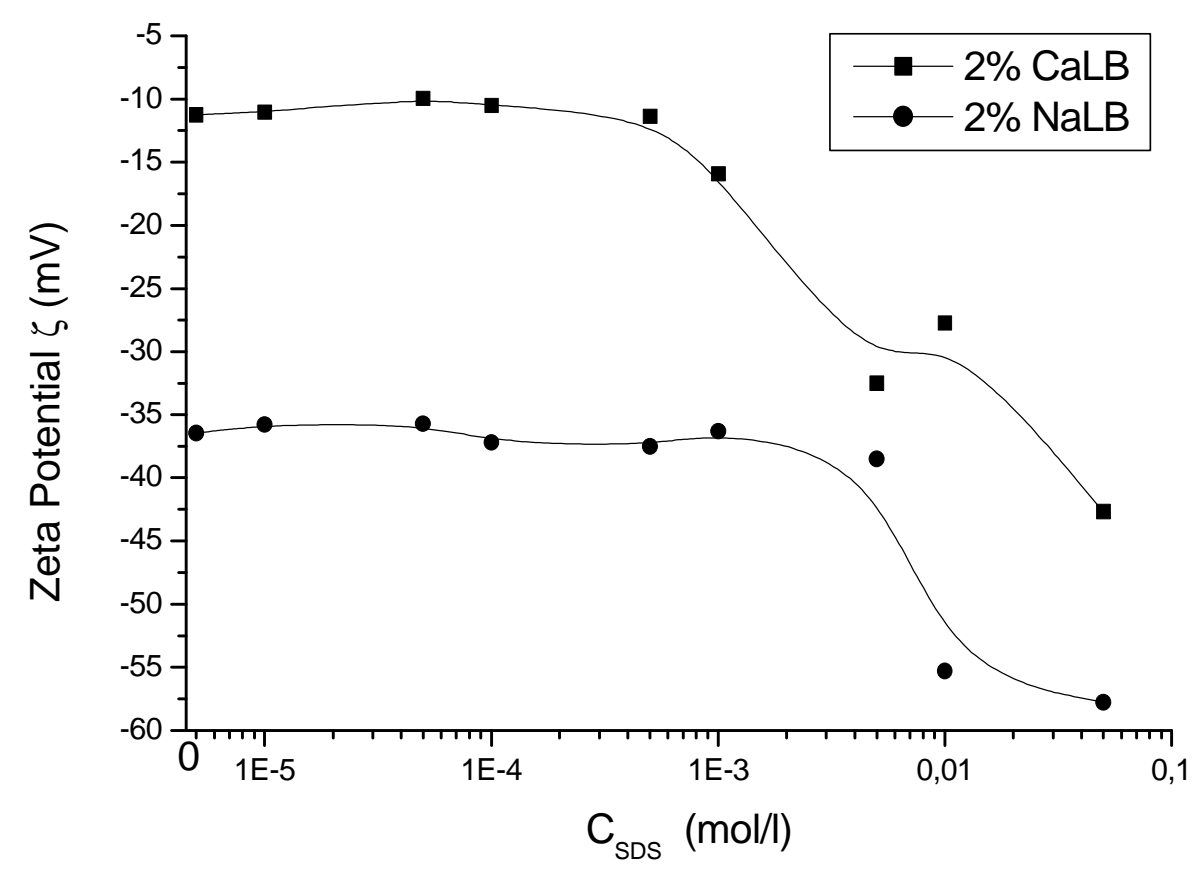

Figure 4. The changes of the zeta potential of bentonite-water systems with SDS added to dispersions.

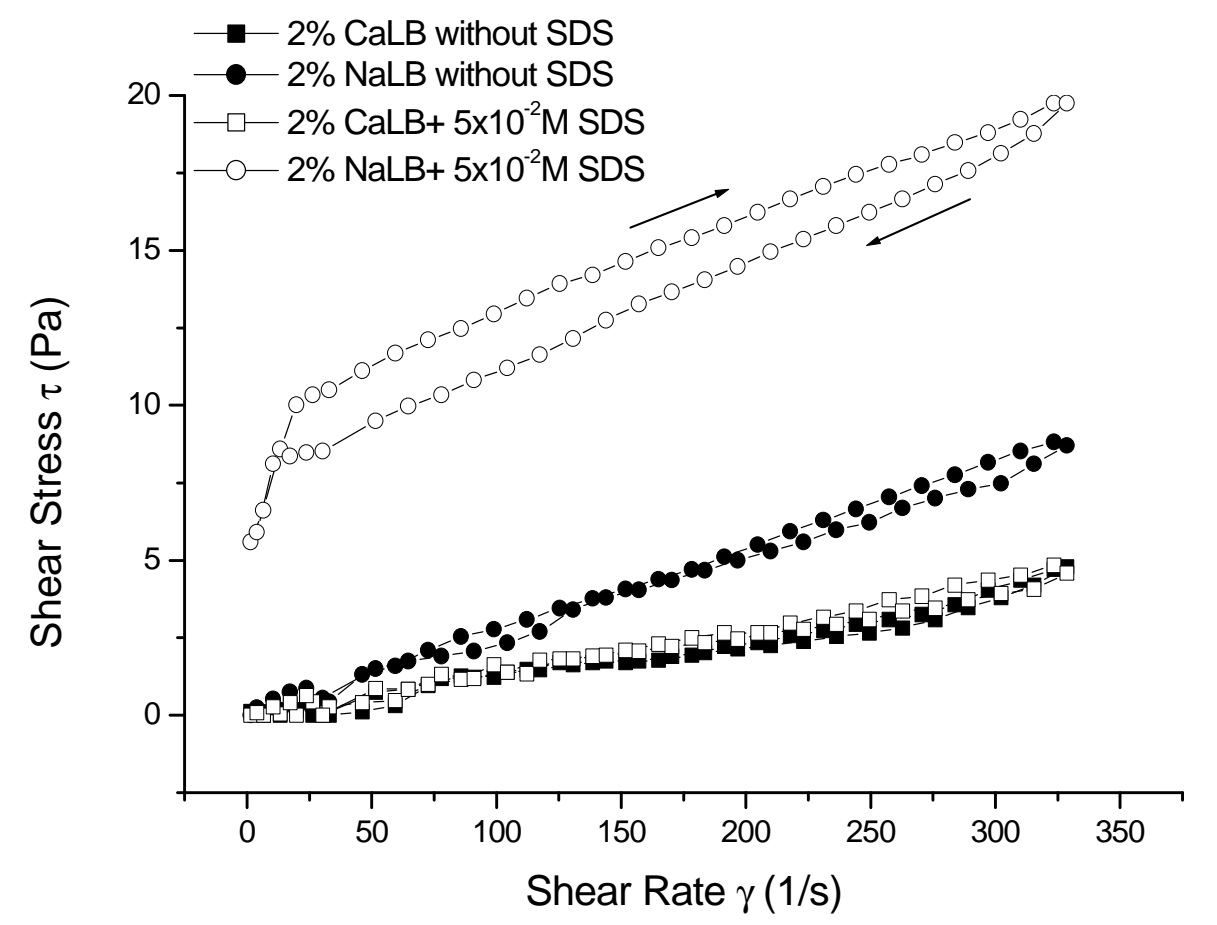

Figure 5. The effect of SDS on flow behaviour of the Ca and Na-bentonite dispersions. 


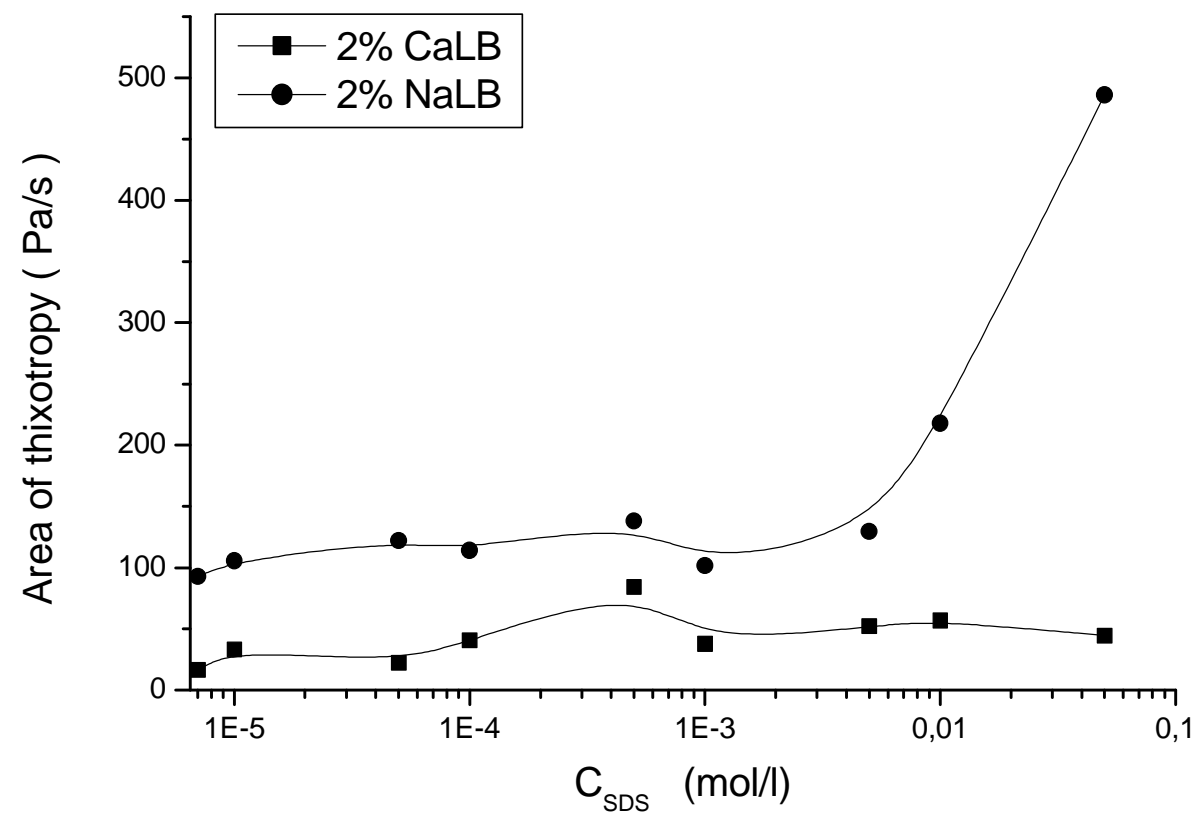

Figure 6. The hysteresis loop area of $2 \% \mathrm{w} / \mathrm{w}$ bentonite dispersion with SDS concentration.

gel appears to be broken by shear forces and the interparticle bonds tend to reestablish themselves with time (Lagaly 1989; Güven 1992). Thixotropy is an important characteristic for industrial applications. For example, drilling fluids and paints must be thixotropic. Bentonite suspensions are thixotropic and, for that reason, have a wide range of industrial applications.

The degree of thixotropic or antithixotropic behaviour was measured by the area of the hysteresis loop (figure 6). These results suggest that NaLB dispersions are more thixotropic than CaLB dispersions. The NaLB dispersions showed much stronger thixotropy after the addition of $10^{-3} \mathrm{~mol} / \mathrm{l}$ of SDS.

\section{Conclusions}

The results show that the plastic viscosity and zeta potential values of $\mathrm{Na}$ activated bentonite dispersions are affected by the addition of anionic surfactant, SDS. The change in rheological properties with effect of SDS is apparently much more stronger for the activated clay (NaLB) compared to the neutral one. The degree of interaction between SDS and the bentonite particles depends on the kind of exchangeable cations and the SDS concentration in the suspension. At SDS concentrations between $5 \times 10^{-4}$ and $10^{-2} \mathrm{~mol} / 1$ the viscosity value of the dispersed NaLB increased to a sharp maximum and then decreased at higher concentrations. SDS enhanced the thixotropy of Na-activated bentonite dispersion. The re- sults indicate that controlled addition of dispersions will result in desired system rheology.

\section{Acknowledgement}

The Research Fund of Istanbul Technical University supported this project.

\section{References}

Alemdar A, Güngör N and Erim F B 2003 Mater. Sci. Letts 22 89

Güngör N 2000 J. Appl. Polym. Sci. 75107

Güngör N, Alemdar A, Atici O and Ece O I 2001 Mater. Lett. 51220

Güven N C M S 1992 Workshop Lectures, Clay Minerals Soc. Boulder

Janek M and Lagaly G 2003 Colloid. Polym. Sci. 281293

Lagaly G 1989 Appl. Clay Sci. 4105

Luckham P F and Rossi S 1999 Adv. Colloid \& Interface Sci. 8243

Michaels A S and Morelos O 1955 Ind. Eng. Chem. 471801

Olphen H Van 1977 Clay colloid chemistry (New York: Wiley)

Penner D and Lagaly G 2000 Clays and Clay Miner. 48246

Welzen J T A M, Stein H N, Stevels J M and Siskens C A M 1981 J. Colloid \& Interface Sci. 81455

Yalçin T, Alemdar A, Ece O I, Güngör N and Çoban F 2002a Mater. Lett. 53211

Yalçin T, Alemdar A, Ece O I and Güngör N 2002b Mater. Lett. 57420 